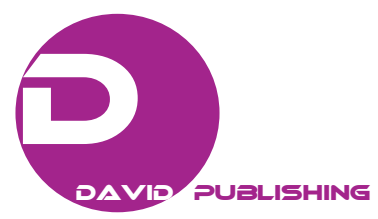

\title{
Numerical Simulation and Kinetic Analysis of Turbine
}

\section{Sail}

\author{
Hu Yihuai, Wang Taiyou and Luan Yongli \\ Department of Marine Engineering, Shanghai Maritime University, 1550, Haigang Avenue, Lingang New City, Pudong New Area, \\ Shanghai, China
}

Received: November 14, 2016 / Accepted: November 22, 2016 / Published: February 28, 2017.

\begin{abstract}
This paper firstly introduces the structure and working principle of turbine sail. Numerical model of a turbine sail is established with Gambit software. The aerodynamic characteristics of the turbine sail are described with RNG $k$ - $\varepsilon$ turbulence model and the numerical simulation is carried out with Fluent software. The influence of sail's structure is analyzed including plate, separation type and height/width ratio. The lift coefficients and drag coefficients of the simulated turbine sail are calculated under different rotation angles, suction intensity and separation plate position. The calculated results are compared with the wind tunnel experimental results, which verifies the feasibility of the numerical results and establishes a foundation for the optimal design of turbine sails.
\end{abstract}

Key words: Turbine sail, aerodynamic characteristics, fluent calculation, wind tunnel test.

\section{Introduction}

Ninety percent goods in the world today is transferred by ships, whose main propulsion power device is still diesel engines. With increasingly consumed global energy resources and more demanding marine environment protection regulations on ship emissions, ship sail-assisting technology has become a hot spot research in shipping and ship building enterprises. Working efficiency of traditional sail is not high enough in limited application scope. The aerodynamic performance of modern wing sail is quite good with higher working efficiency, but it takes up too much space on board ship [1]. Turbine sail has high energy saving efficiency and occupies small space. By extracting air flow into the sail its lift coefficient could reach 6.5 to 7.5. In 1980s, a French research group carried out a wide range of research work including a number of wind tunnel experiments and tests on the ALCYONE ship. The energy saving

Corresponding author: $\mathrm{Hu}$ Yihuai, Ph.D., professor, research fields: mechanical fault diagnosis and new engery application on board ship. efficiency of turbine sail could be about $50 \%$ under a favourable wind velocity of $12.8 \mathrm{~m} / \mathrm{s}$, much higher than that of the traditional and wing sails [2]. However, little research work has been carried out on the turbine sail by now.

Many factors could affect turbine sail performance such as type, position, height/width ratio of separation plate, rotation angle, suction intensity of elliptical cylinder [3]. Under different wind conditions, the position of separation plate, rotation angle of elliptical cylinder and suction intensity could be adjusted. But the type and height/width ratio of separation plate could not be changed once the turbine sail is built up. This paper makes the simulation of a typical turbine sail with Fluent software and analyzes the influential factors including type, position, and height/width ratio of separation plate and rotation angle, suction intensity of elliptical cylinder on the aerodynamic performance of turbine sail.

\section{Working Principle of Turbo Sail}

A streamlined object will be subjected to drag force resulted from shedding vortex behind the object. The 
worse the streamline is, the more easily the vortex sheds and the larger the drag force will be. But there will be not any lift force induced. If flow is sucked near the separation point to delay or stop the flow separation, it will prevent the shedding vortex and induce both lift and drag forces. This is the working principle of turbine sail [4] as indicated in Fig. 1.

Generally, the separation of boundary layer could be divided into two types, one occurs on the smooth surface, the other occurs at sharp corners or other discontinuities of the surface [5]. The separation on smooth surface is mainly due to the lack of energy in the boundary layer, because the boundary layer fluid must overcome the viscous drag when flowing, which will result in the continuous loss of momentum. Boundary layer flow can only be separated from the surface at the speed of zero, forming a vortex. At the corners, the flow speed is very small and the pressure is very low, which directly causes high inverse pressure gradient in downstream flow, so these mainly leads to the separation [6].

The turbine sail uses flow suction method to control boundary layer separation and its structure is shown in Fig. 2. The main structure of turbine sail is a rotatable elliptical cylinder with small holes on two sides of the trailing edge. A movable separation plate is installed to adjust flow direction on the surface, which is designed in the shape of rear airfoil to reduce the vortex intensity near the suction surface. The upper and lower ends of the elliptic cylinder are equipped with endplates to secure stability and an air pump is installed under the upper endplate. Here the separation plate could change the air flow and the flow separation position. The suction pump could provide energy for the flow in boundary layer. The combined effect of these two devices could apparently improve the aerodynamic performance of turbine sail, and thereby induces a kind of lift force. If wind velocity and wind, direction changes the position of separation plate, rotation angle of elliptical cylinder and pumped suction flow could be adjusted to achieve the best propulsion performance of the turbine sail.

When the turbine sail is installed on a ship, its lift force $F_{\mathrm{L}}$ and drag force $F_{\mathrm{D}}$ acting on the sail are shown in Fig. 3. Where $\mathrm{V}$ is direction of apparent wind; $\mathrm{L}$ is the direction of lift force; $\mathrm{D}$ is the direction of drag force; $\varphi$ is the angle between apparent wind direction and ship navigation direction; $\theta$ is rotation angle between elliptical cylinder major axis and ship navigation direction, which is positive in anticlockwise. $\lambda$ is rotation angle of separation plate from separation

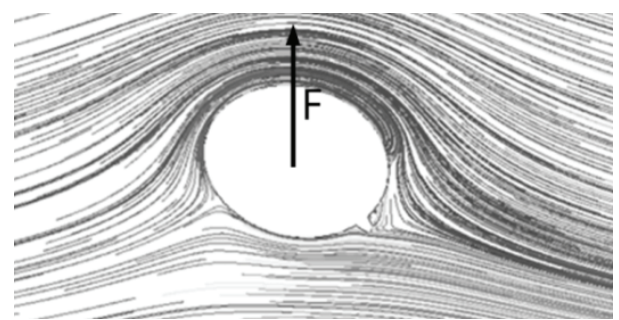

Fig. 1 Working principle of turbine sail.

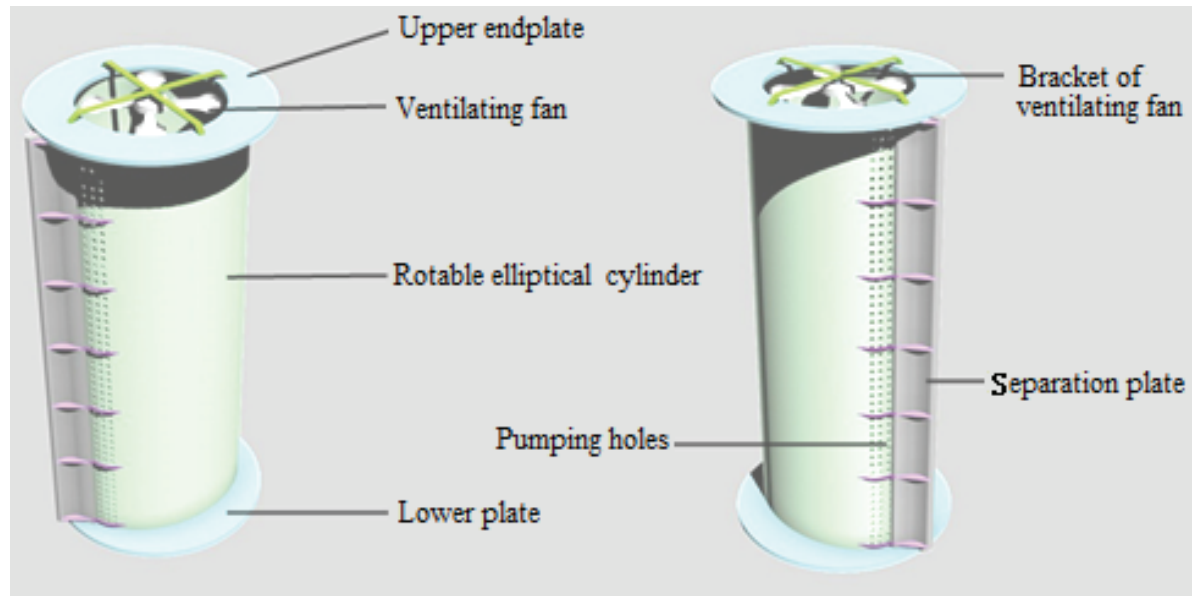

Fig. 2 Structure diagram of turbine sail. 


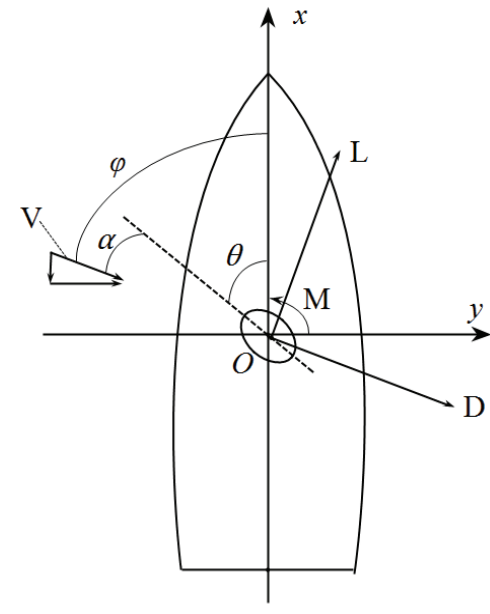

Fig. 3 Forces acting on the sail in wind coordinates.

plate centerline to elliptical cylinder major axis, which is positive in count-clockwise. The life and drag forces of turbine sail could be described in terms of dimensionless parameters as:

$$
\begin{aligned}
C_{L} & =F_{L} / \frac{1}{2} \rho S V^{2} \\
C_{D} & =F_{D} / \frac{1}{2} \rho S V^{2}
\end{aligned}
$$

where,

$$
\begin{aligned}
& \rho \text {-Air density }\left(\mathrm{kg} / \mathrm{m}^{3}\right) ; \\
& S \text {-Surface area of elliptical cylinder }\left(\mathrm{m}^{3}\right) ; \\
& V \text {-Apparent wind velocity }(\mathrm{m} / \mathrm{s}) .
\end{aligned}
$$

\section{Numerical Modeling and Analysis}

The suction holes are distributed along $20^{\circ} \sim 30^{\circ}$ arc and the circumferential moving range of separation plate along the elliptical cylinder is $20^{\circ} \sim$ $30^{\circ}$ arc length. The length and width of calculation zone are 100 times and 20 times of the ellipse short axis respectively with about $2.2 \times 10^{6}$ grids. The velocity_inlet type is taken as the entrance of calculation zone with inflow wind velocity. The out flow type is used at the exit of the zone with free flow. The quantity_inlet type is taken as the suction side and the solid wall condition type is used on the other surface of boundary.

The numerical simulation was made with simple algorithm and RNG $k-\varepsilon$ turbulence model. In order to improve the calculation accuracy, a second order upwind scheme is adopted [3]. Some simplifications are made as:

- The temperature influence is ignored.

- The area of suction holes is equal to that of suction surface.

- The wind pressure gradient is ignored.

The RNG $k$ - $\varepsilon$ turbulence model [7] is used because of the suction effect, circulating flow around elliptical cylinder and vortex around the sail:

$$
\frac{\partial(\rho k)}{\partial t}+\frac{\partial\left(\rho k u_{i}\right)}{\partial x_{i}}=\frac{\partial}{\partial x_{j}}\left[\alpha_{k} \mu_{e f f} \frac{\partial k}{\partial x_{j}}\right]+G_{k}+\rho \varepsilon
$$

$$
\frac{\partial(\rho \varepsilon)}{\partial t}+\frac{\partial\left(\rho \varepsilon u_{i}\right)}{\partial x_{i}}=\frac{\partial}{\partial x_{j}}\left[\alpha_{\varepsilon} \mu_{e f f} \frac{\partial \varepsilon}{\partial x_{j}}\right]+\frac{C_{l \varepsilon} \varepsilon}{k} G_{k}-C_{2 \varepsilon} \rho \frac{\varepsilon^{2}}{k}
$$

where, $\mu_{e f f}=\mu+\mu_{t}, \quad \mu_{t}=C_{\mu} \rho \frac{k^{2}}{\varepsilon}, \quad C_{\mu}=0.0845$, $\alpha_{k}=\alpha_{\varepsilon}=1.39, C_{1 \varepsilon}=1.42, C_{2 \varepsilon}=1.68$.

The turbulence intensity $I$, turbulence kinetic energy $k$ and turbulent dissipation rate $\varepsilon$ are calculated as:

$$
I=\mu^{\prime} / \bar{\mu}=0.16\left(\operatorname{Re}_{D H}\right)^{-1 / 8}
$$

$$
k=\frac{3}{2}(\bar{\mu} I)^{2}
$$

$$
\varepsilon=C_{\mu} 3 / 4 \frac{k^{3 / 2}}{l}=\rho C_{\mu} \frac{k^{2}}{\mu}\left(\frac{\mu_{t}}{\mu}\right)^{-1}
$$

Here $C_{\mu}=0.09, \mu_{t} / \mu$ is turbulent viscosity ratio, $\mu^{\prime}$ is turbulent fluctuation velocity, $\bar{\mu}$ is average speed, $\operatorname{Re}_{D H}$ is Reynolds number calculated from hydraulic diameter $D_{H}$.

A concept of suction intensity $\gamma$ is introduced to indicate the suction condition as the ratio of inflow velocity through suction surface to velocity at infinity:

$$
\gamma=\frac{V_{\infty}}{V_{i n}}
$$

The working efficiency of turbine sail could be greatly influenced by its structural parameter including rotation angle of elliptical cylinder, suction 
intensity and separation plate position.

\subsection{Rotation Angle of Elliptical Cylinder}

Under the condition of $10 \mathrm{~m} / \mathrm{s}$ wind velocity, 1.5 suction intensity and different rotation angle from elliptical cylinder from $-40^{\circ}$ to $30^{\circ}$ by $5^{\circ}$ step, some simulated streamlines in the trailing edge of elliptical cylinder are shown in Fig. 4. When under $-40^{\circ}$, vortex in the trailing edge of elliptical cylinder will shed alternately presenting unstable flow field, resulting in unstable lift and drag coefficients of the sail. This shedding vortex may induce structural vibration and harmful resonance endangering sail structure. When $\theta$ is between $-30^{\circ}$ and $5^{\circ}$, the vortex will disappear presenting stable flow field and resulting in large lift coefficients, which is believed to be the ideal working condition of the sail.

When $\theta$ is between $15^{\circ}$ and $30^{\circ}$, single vortex will be formed in the trailing edge of elliptical cylinder without shedding and the flow field will tend to be stable but the lift coefficients will be quite smaller. When under $-15^{\circ}$, the lift coefficient will get to its maximum and the drag coefficient will be very small as shown in Fig. 5. So the available rotation angle of elliptical cylinder for ship propulsion is between $-15^{\circ}$ and $0^{\circ}$.

\subsection{Shape of Separation Plate}

Two simulations are carried out under the condition of $10 \mathrm{~m} / \mathrm{s}$ wind velocity, $0^{\circ}$ rotation angle of elliptical cylinder and 1.6 suction intensity of turbine sail. One is with a streamlined separation plate and the other is without separation plate. In the case of no separation plate, stable flow field could not be formed in the trailing edge of elliptical cylinder and serials of vortex will shed alternately. Due to the influence of shedding vortex, the lift and drag coefficients of turbine sail fluctuate periodically inducing noise and vibration and worsening the stability of turbine sail [8]. In case of separation plate, the vortex disappears in trailing edge of elliptical cylinder and the flow field is tending to be

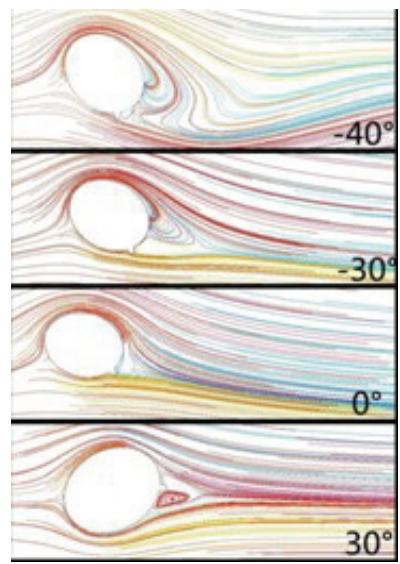

Fig. 4 Streamlines with different rotation angle of elliptical cylinder.

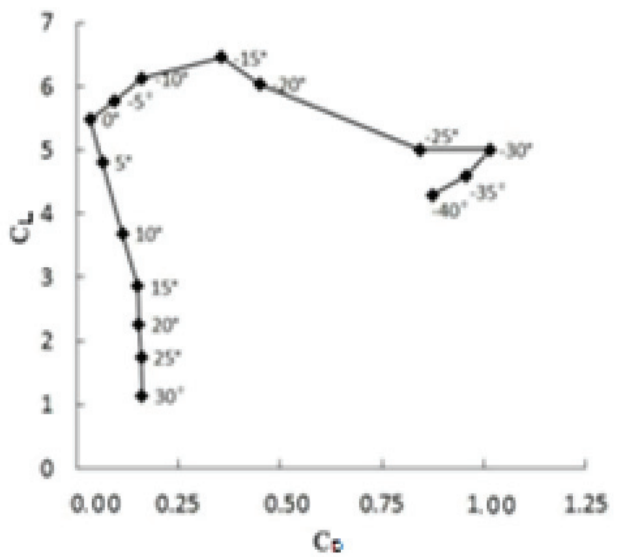

Fig. 5 Lift and drag coefficients under different rotation angles.

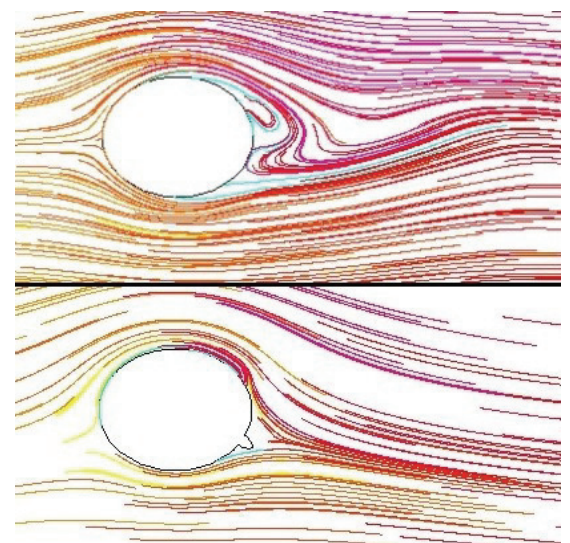

Fig. 6 Streamline with or without separation plate.

smooth, which greatly improves lift coefficient as indicated in Fig. 6.

Another two simulations are carried out under the conditions of $10 \mathrm{~m} / \mathrm{s}$ wind velocity, $0^{\circ}$ rotation angle of elliptical cylinder, 0.6 suction intensity of turbine 


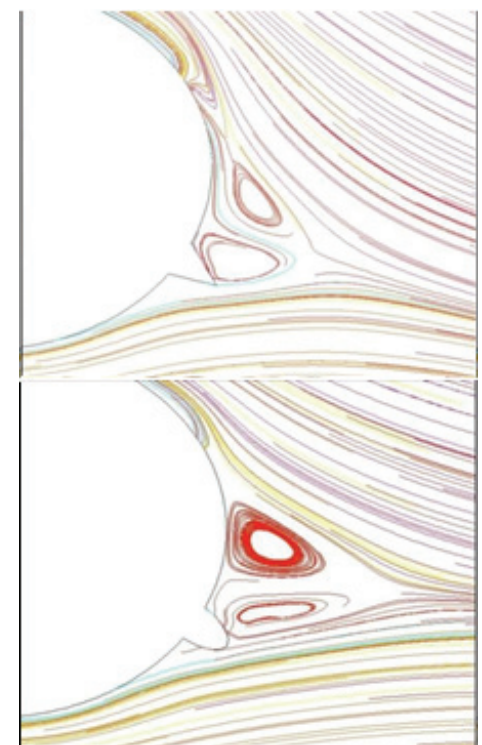

Fig. 7 Separation point of different types of separation plate.

sail and 1.25 height/width ratio of separation plate. The streamlines in the trailing edge of elliptical cylinder with streamlined and sharp shaped separation plate are shown respectively in Fig. 7. It is indicated that the separation position of sharp shaped separation plate is almost at the sharp corner, while the separation position of streamlined separation plate is in the downstream.

Under the same condition but with different suction intensity from 0.2 to 2.4 at 0.2 step, the lift and drag coefficient of the sail are calculated as shown in Fig. 8. The lift coefficient is higher and the drag coefficient is lower of the sail with streamlined separation plate when the suction intensity is larger than 0.2 . When suction intensity is larger than 0.8 , vortex in the trailing edge of elliptical cylinder with streamlined separation plate will disappear presenting stable flow field. But vortex in the trailing edge of elliptical cylinder with sharp shaped separation plate does not disappear, even the flow field tends to be stable without shedding vortex. It is concluded that the streamlined separation plate has better aerodynamic performance than the sharp shaped separation plate.

\subsection{Height/Width Ratio of Separation Plate}

With $10 \mathrm{~m} / \mathrm{s}$ wind velocity, $0^{\circ}$ rotation angle of the elliptical cylinder, different height/width ratio of separation plate as $0.5,1.25,2.0,2.75$ and different suction intensity from 0.2 to 2.4 by 0.2 step, the lift coefficients and drag coefficients are calculated as shown in Fig. 9. As seen the lift coefficients increase more greatly with suction intensity than the drag coefficients do, but they all increase with height/width ratio of separation plate when suction intensity is 0.2 . This is because that height/width ratio of separation plate could supply more energy even under weak suction intensity of the sail. But too large height/width ratio could increase not only the drag coefficient, but also the moment on separation plate as well. So the best height/width ratio of separation plate is 2.0 .

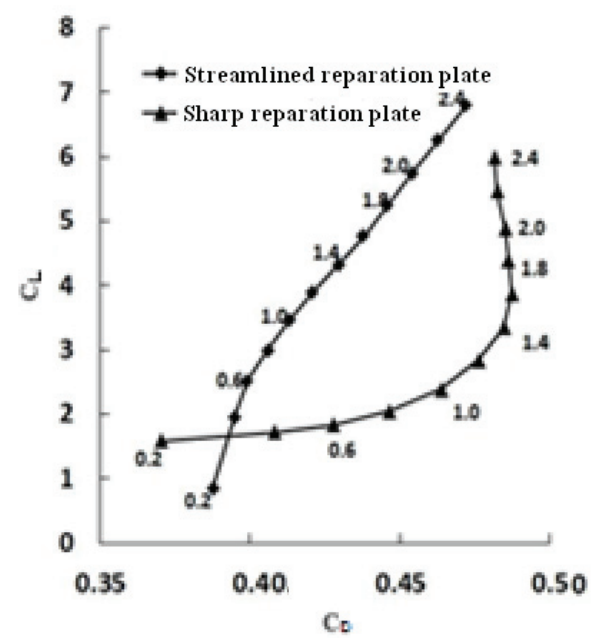

Fig. 8 Lift and drag coefficients with different types of separation plate.

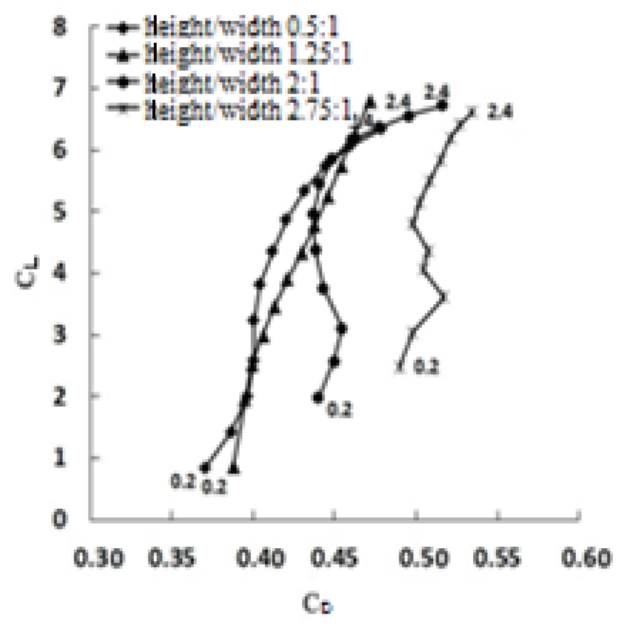

Fig. 9 Lift and drag coefficients with different height/width ratio and different suction intensity. 


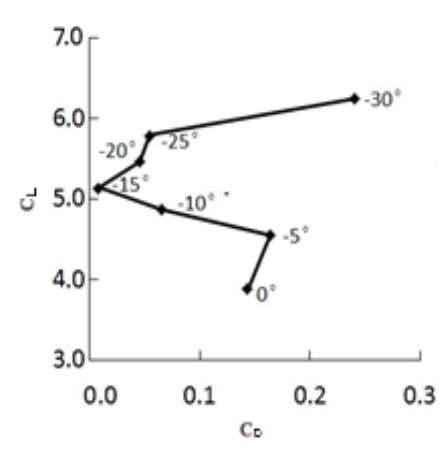

Fig. 10 Lift and drag coefficients with different rotation angle of separation plate.

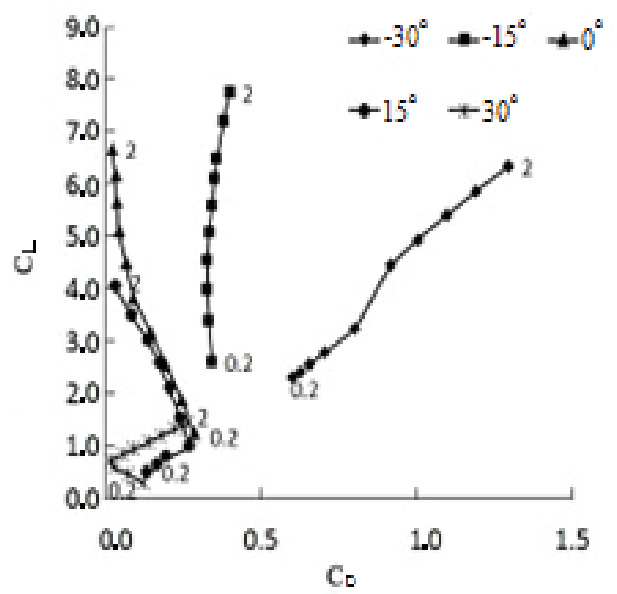

Fig. 11 Lift and drag coefficients with different suction intensity and elliptical cylinder rotation angle.

\subsection{Position of Separation Plate}

The lift and drag coefficients are calculated under $10 \mathrm{~m} / \mathrm{s}$ wind velocity, 1.5 suction intensity, $0^{\circ}$ rotation angle of turbine sail and different rotation angle of separation plate from $0^{\circ}$ to $-30^{\circ}$ by step $5^{\circ}$ as seen in Fig. 10. It is found that lift coefficient increases with rotation angle of separation plate $\lambda$, while drag coefficient does not change regularly. When rotation angle of separation plate is between $-15^{\circ}$ and $-30^{\circ}$, the vortex will disappear and the flow field will become smooth and stable.

\subsection{Suction Intensity}

When suction intensity ranges from 0.2 to 2 at 0.2 step, the lift and drag coefficients are calculated with different rotation angle of turbine sail as shown in Fig. 11. It is seen in Fig. 9 that the lift coefficient increases with suction intensity under different rotation angle of elliptical cylinder. Lift coefficients are larger than 6.0 when suction intensity is stronger than 1.8 and $\theta$ is $-30^{\circ},-15^{\circ}$ and $0^{\circ}$ respectively. The drag coefficient increases with suction intensity greatly when $\theta$ is $-30^{\circ}$. It increases slightly when $\theta$ is $-15^{\circ}$ and decreases when $\theta$ is $0^{\circ}$ with increasing suction intensity. When $\theta$ is $15^{\circ}$ and $-30^{\circ}$ the lift and drag coefficients are both quite small under different suction intensity without any practical use.

From the simulated streamlines with different suction intensity it is found that when $\gamma<0.4$ the shedding vortex in the trailing edge of elliptical cylinder will alternately occur under all the rotation angles. When $\gamma$ equals 0.6 , a single stable vortex will appear under $0^{\circ}$ rotation angle of elliptical cylinder. When $\gamma>0.8$ a single stable vortex will occur in the trailing edge only when the rotation angle of elliptical cylinder is between $-15^{\circ}$ and $\theta=-30^{\circ}$. The reason for the shedding vortex in the trailing edge of elliptical cylinder when $\gamma<0.4$ may be due to the low provided energy for boundary flow. The optimal suction intensity is between 1.2 and 1.8 when air pump efficiency, lift/drag coefficients and turbine sail stability are taken into account.

\section{Wind Tunnel Test}

Under the conditions of $10 \mathrm{~m} / \mathrm{s}$ wind velocity, $-30^{\circ}$ rotation angle of elliptical cylinder, 1.25 height/width ratio and streamlined type separation plate, calculated lift coefficient has the same tend with that of wind tunnel experimental results by a French scientific research group that:

(1) The best rotation angle of turbine sail is between $-15^{\circ}$ and $0^{\circ}$;

(2) The optimal suction intensity of turbine sail is from 1.2 and 1.8 .

To verify the simulated results mentioned above experiments are carried out on turbine sail models under $7 \mathrm{~m} / \mathrm{s}$ and $10 \mathrm{~m} / \mathrm{s}$ wind velocity respectively in a low-speed wind tunnel laboratory of Shanghai Maritime 


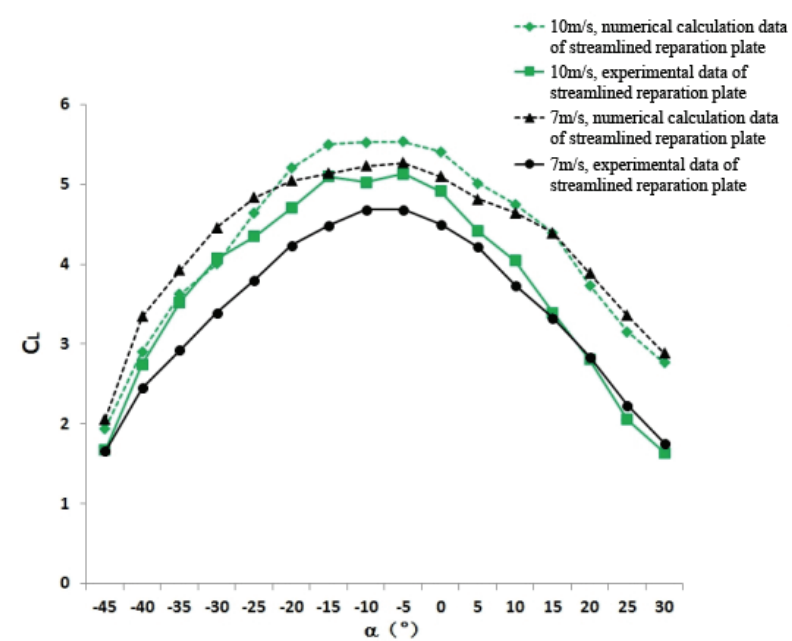

Fig. 12 Lift coefficients with streamlined separation plate.

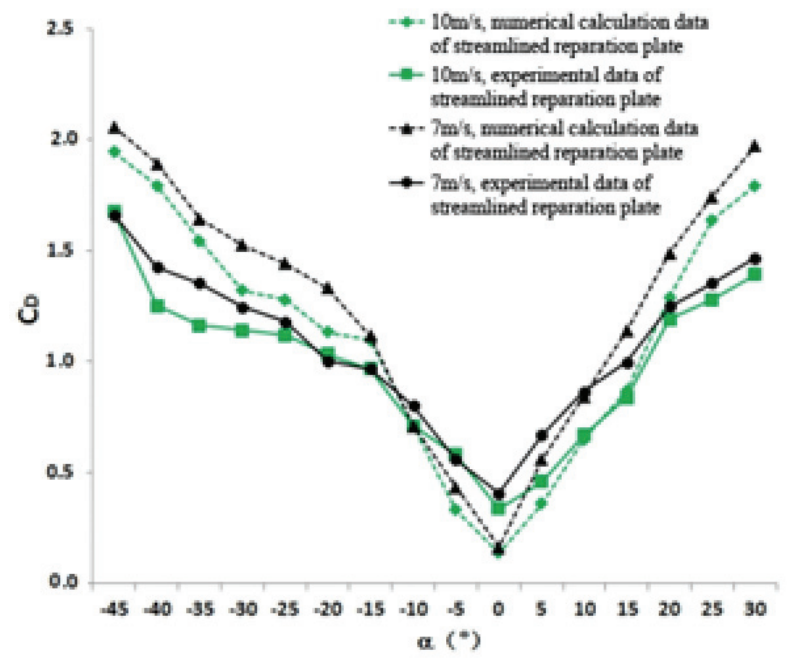

Fig. 13 Drag coefficients with streamlined separation plate.

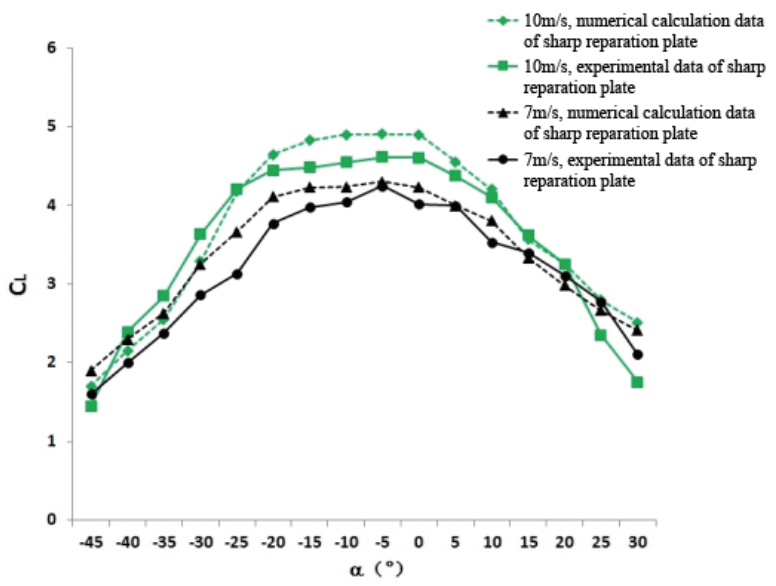

Fig. 14 Lift coefficients with sharp shaped separation plate.

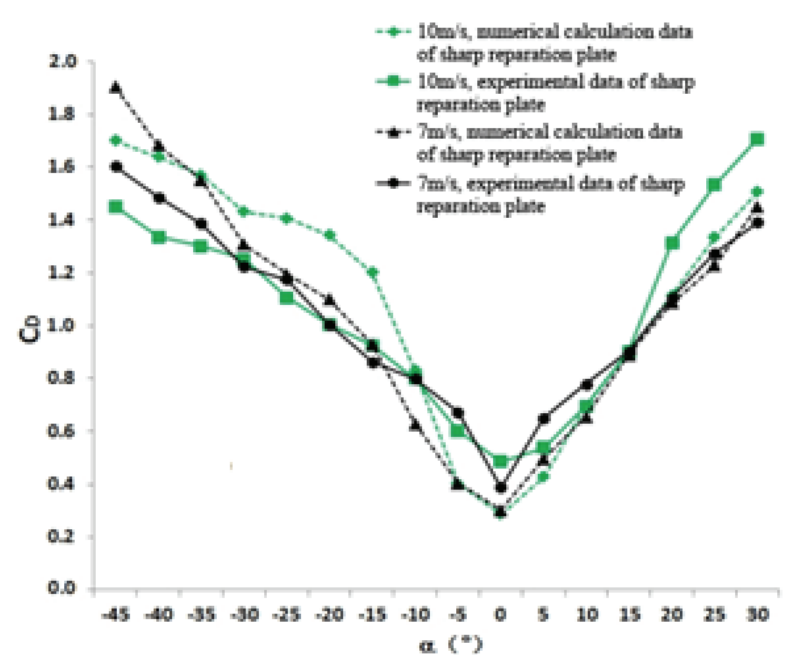

Fig. 15 Drag coefficients with sharp shaped separation plate.

University. Its wind velocity ranges from $1.0 \mathrm{~m} / \mathrm{s}$ to $40.0 \mathrm{~m} / \mathrm{s}$ and the measuring accuracy is $\pm 0.1 \%$ (FS). The measured forces are transformed into lift/drag coefficients and compared with simulated results as shown in Figs. 12-15 respectively.

As seen in Figs. 12 and 13, the simulated lift coefficients with streamlined separation plate are larger than the experimental results under both $10 \mathrm{~m} / \mathrm{s}$ and $7 \mathrm{~m} / \mathrm{s}$ wind velocities. The simulated drag coefficients are larger than experimental results when attack angle of turbine sail $a$ ranges from $-45^{\circ}$ to $-15^{\circ}$ and from $15^{\circ}$ to $30^{\circ}$, but smaller than experimental results when attack angle ranges from $-10^{\circ}$ to $10^{\circ}$.

As seen in Figs. 14 and 15, the simulated lift coefficients with sharp shaped separation plate are a little larger than the experimental results under $10 \mathrm{~m} / \mathrm{s}$ wind velocity but more larger under $7 \mathrm{~m} / \mathrm{s}$ wind velocity. The deviation of drag coefficients with sharp shaped separation plate is nearly the same with that with streamlined separation plate when attack angle ranges from $-10^{\circ}$ to $10^{\circ}$. They all have the same trends between simulated and experimental results.

It is concluded that lift coefficients increase and drag coefficients decrease gradually when attack angle of turbine sail ranges from $-45^{\circ}$ to $0^{\circ}$. Then the lift coefficients decrease and drag coefficients increase 
gradually when attack angle of turbine sail ranges from $0^{\circ}$ to $30^{\circ}$. All the numerical simulated results and wind tunnel experimental results have the same varying tendency, which verifies the feasibility of both simulated and experimental results. The variation between these two results may result from the assumptions made for the simulation, model materials and conditions for the tests.

\section{Conclusions}

(1) The application of separation plate could avoid the shedding vortex in the trailing edge of elliptical cylinder and improve the flow field around the elliptical cylinder effectively, which therefore increases the lift coefficient of turbine sail significantly.

(2) The lift coefficients are larger and the drag coefficients are smaller of the turbine sail with streamlined separation plate than that with sharp shaped separation plate and its flow field in the trailing edge of elliptical cylinder is more stable, which indicates the better performance of streamlined separation plate than the sharp shaped separation plate.

(3) The height/width ratio of streamlined separation plate has also influence on the performance of turbine sail. The optimal height/width ratio is 2.0 if the air pump efficiency and forces acting on separation plate are taken into account.

(4) The rotation angle of elliptical cylinder has great influence on the lift/drag coefficient of the sail. Improper rotation angle could not only induce the shedding vortex in the trailing edge of elliptical cylinder and abnormal vibration of turbine sail, but also result in poor performance and low working efficiency of the sail. The optimal rotation angle of the turbine sail is between $-15^{\circ}$ and $0^{\circ}$.

(5) The lift coefficients of turbine sail increase with suction intensity. The most suitable suction intensity is between 1.2 and 1.8 when the air pump efficiency, lift coefficients, drag coefficients, working condition of turbine sail and vortex behind the elliptical cylinder are taken into account.

(6) The rotation angle of separation plate has some influence on the lift/drag coefficient and the vortex in trailing edge. When the rotation angle is between $-15^{\circ}$ and $-30^{\circ}$, the flow field will be relatively stable and the lift coefficient will be relatively larger.

\section{Acknowledgements}

The contributors of this paper include Mr. Zhu Pengxu and Mr. Li Zhiqiu for the assisting of the wind tunnel tests.

This research work is supported by STCSM within the project of "Research on Application of sail on Sea-going ships”, Project Number: 08210511800.

\section{References}

[1] Lu, L. 2011. "Research on Modeling and Simulation of Sail-Assisted Ship's Power Plants.” Degree dissertation, Wuhan: Wuhan University of Technology.

[2] Hu, Y. 2015. New Energy and Ship Energy Saving Technology, 1st ed. Science Press.

[3] Zhang, J. 1986. "The Development and Research of the Wind Power for Merchant Ship in France." Translated Collection of Ship and Ocean Engineering 1 (3): 51-2.

[4] Chen, Z., Jin, C., and Wang, H. et al. 2004. Engineering Fluid Mechanics, 2nd ed. China Higher Education Press.

[5] Li, X. 2007. "Research on the Electromagnetic Force in Cylinder Wake Control in the Lower Reynolds Number." Degree dissertation, Nanjing: Nanjing University of Science and Technology.

[6] Ding, H., Fan, B., Zhou, B., and Chen, Z. 2005. "Boundary Layer of Flow Around Cylinder and Its Electromagnetic Control." Journal of Nanjing University of Science and Technology 29 (3): 308-11.

[7] Zhang, S. 2011. Computational Fluid Dynamics and Its Application - The Principles and Applications of CFD Software, 1st ed. Huazhong University of Science \& Technology Press.

[8] Liu, A. 1999. "The Development and Existing Problems of Sail-Assisted Ship Technology." Marine Energy Saving 13 (2): 8-12.

[9] Tong, B., Yi, X., and Zhu, K. 2009. Vortex Motion Theory. University of Science and Technology China Press. 